\title{
Buehler 置信限的计算*
}

\author{
房祥忠 ${ }^{* *}$ 陈家鼎
}

(北京大学数学学院, 北京大学数学与应用数学教育部开放实验室, 北京 100871)

\begin{abstract}
摘要在医学研究和产品研制过程中, 由于试验对象难于找到或者试验费用 昂贵常出现小样本情形. 此时, 精确置信推断尤其重要. 只要在样本空间中给出 一种序就可以定义模型参数的某个函数的精确置信限. 这样得到的置信限称为 Buehler 置信限. 虽然它的定义比较容易, 但是当多维参数或者不完全观测数据 出现时, 计算有时难于实行. 为了解决这种计算问题, 本文构造出一种基于 EM 算法的方法. EM 算法原本是用于求解极大似然估计的方法，在这里 EM 算法首 次被用于求解精确置信限. 分析了 3 种模型和一组实际数据以说明这个方法.
\end{abstract}

\section{关键词 Buehler 置信限 EM 算法 小样本}

统计中的一个重要的问题是对于模型中参数的某个感兴趣的函数给出好的 置信限. 构造精确置信限的一个一般方法是 Buehler 的方法. Buehler ${ }^{[1]}$ 给出这种 方法构造二项分布等离散分布中参数函数的置信限. Jobe 等 ${ }^{[2]}$ 和陈家鼎 ${ }^{[3]}$ 将这 种方法推广到相当一般的模型. 尽管这种方法目前主要用于可靠性中，但是它 应该有更加广泛的应用. 按照这种一般理论，如果在样本空间中定义一种序，则 对于任何参数的实值函数总可以定义出一种关于序单调的精确置信下限 (LCL) 和置信上限 (UCL), 而且这种置信限在关于序单调的所有置信限中是最优的. 同 时，对任何一个精确置信限，总可以通过这种方法构造一种不次于所给置信限 的精确置信限. 通常情形下, 给定函数的一个点估计用来定义样本空间的序，但 也有其他选择 (参见文献 [4]). 虽然它的定义比较容易, 但是当多维参数或者不完 全观测数据出现时, 计算有时难于实行. 为了解决这样的计算问题, 我们在本文 给出一种基于 EM 算法的方法.

$\mathrm{EM}$ 算法是由 Dempster 等人 ${ }^{[5]}$ 给出用来寻求在缺失数据或者不完全数据情 形下极大似然估计 (MLE) 或者后验分布极值点的方法. 为了使用方便和增加收 
玫速度又出现了许多 EM 型算法, 如期望条件最大化选择算法 (ECME) ${ }^{[6]}$ 和交互 期望条件极大化方法 (AECM) ${ }^{[7]}$. 所有这些发展促使我们将求精确置信限的问题 转化成求解最大似然估计的问题，从而可以使用各种 EM 算法解决 Buehler 置信 限的计算问题，这是 $\mathrm{EM}$ 算法首次用于求解精确置信限.

\section{Buehler 置信限和它的新型式}

假定观测值 $z \in E$ 来自随机变量 $Z$, 而 $Z$ 服从概率分布 $P_{\theta}$, 这里 $\theta$ 在集合 $\Theta$ 中取值. 称 $E$ 为样本空间. 设 $g(\theta)$ 是 $\Theta$ 上的一个实值函数. 我们的目标是寻求 $g(\theta)$ 的精确置信下限或置信上限.

假如在样本空间 $E$ 中有一个全序关系 “ $\succeq$ ”, 则对任意 $z \in E, \theta \in \Theta, \alpha \in$ $(0,1)$, 设

$$
\begin{gathered}
G(z, \theta)=P_{\theta}(Z \succeq z), \\
H(z, \theta)=P_{\theta}(Z \succ z), \\
\underline{g}(z)=\inf \{g(\theta): \theta \in \Theta, G(z, \theta)>\alpha\}, \\
\bar{g}(z)=\sup \{g(\theta): \theta \in \Theta, H(z, \theta)<1-\alpha\},
\end{gathered}
$$

这里 $\inf \emptyset=\sup \{g(\theta): \theta \in \Theta\}, \sup \emptyset=\inf \{g(\theta): \theta \in \Theta\}$. 本文符号 $\emptyset$ 表示空集.

陈家鼎 ${ }^{[3]}$ 给出了一个正规化的条件: 样本空间 $E$ 有一个可数子集 $E_{1}$, 对于 任意 $a, b \in E$ 和 $a \succ b$, 存在 $c \in E_{1}$, 使得 $a \succeq c$ 并且 $c \succeq b$. 在这个正规化的条件下 给出如下的结论:

引理 1.1 在正规化条件下, 对于所有的 $\alpha \in(0,1), \theta \in \Theta$, 下面两个不等式 成立:

$$
\begin{aligned}
& P_{\theta}(g(\theta) \geqslant \underline{g}(Z)) \geqslant 1-\alpha, \\
& P_{\theta}(g(\theta) \leqslant \bar{g}(Z)) \geqslant 1-\alpha .
\end{aligned}
$$

即 $\underline{g}(Z)(\bar{g}(Z))$ 是 $g(\theta)$ 的置信下 (上) 限, 置信水平为 $1-\alpha$. 另外, $\underline{g}(Z)(\bar{g}(Z))$ 在 所有关于序单调且具有同样水平的置信下 (上) 限中是最大 (小) 的.

因为 $G(z, \theta)$ 和 $H(z, \theta)$ 通常都是 $\theta$ 的比较复杂的函数, 在 (1.3) 和 (1.4) 式 中的最优值不容易得到. 尽管对于每一个 $\theta$ 可以利用 Monte Carlo 模拟技术计算 $G(z, \theta)$ 和 $H(z, \theta)$, 但是我们仍然不知道从哪个方向出发寻求最优值. 为了得到 这些最优值, 我们将这个问题变换成它的对偶问题, 然后用 EM算法解决它. 对 任意实数 $c$, 定义

$$
\begin{gathered}
\bar{G}(z, c)=\sup \{G(z, \theta): g(\theta)<c\}, \\
\bar{H}(z, c)=\sup \{1-H(z, \theta): g(\theta)>c\},
\end{gathered}
$$

这里 $\inf \emptyset=\sup \{G(z, \theta): \theta \in \Theta\}, \sup \emptyset=\inf \{1-H(z, \theta): \theta \in \Theta\}$, 则我们可以 得到下面的定理： 
定理 1.1 在和引理 1.1 同样的条件下, 如果 $\underline{g}(z)$ 和 $\bar{g}(z)$ 由 (1.3) 和 (1.4) 式 定义, 则下面的两个等式成立:

$$
\begin{aligned}
& \underline{g}(z)=\sup \left\{c: \bar{G}(z, c) \leqslant \alpha, c_{0} \leqslant c \leqslant c_{1}\right\}, \\
& \bar{g}(z)=\inf \left\{c: \bar{H}(z, c) \leqslant \alpha, c_{0} \leqslant c \leqslant c_{1}\right\},
\end{aligned}
$$

这里 $c_{0}=\inf \{g(\theta): \theta \in \Theta\}, c_{1}=\sup \{g(\theta): \theta \in \Theta\}, \sup \emptyset=c_{0}, \inf \emptyset=c_{1}$.

证明比较容易, 故略去.

由于 $\bar{G}(z, c)$ 单调递增并且关于 $c$ 左连续, $\bar{H}(z, c)$ 单调递减并且关于 $c$ 右 连续, 如果对于任意 $c$ 它们的值可以得到，则可以用二分法，分别通过 (1.9) 和 (1.10) 式求出 $\underline{g}(z)$ 和 $\bar{g}(z)$, 因此难点只在于如何得到极值 $\bar{G}(z, c)$ 和 $\bar{H}(z, c)$.

如果 $G(z, \theta)$ 和 $g(\theta)$ 对于 $\theta$ 的某些分量是连续的, 则 (1.7) 式中的限制条件 $g(\theta)<c$ 可以被 $g(\theta) \leqslant c$ 替代. 更进一步，如果 $G(z, \theta)$ 和 $g(\theta)$ 是单调的并且最 大值可以在 $g(\theta) \leqslant c$ 的边界上得到，则限制条件可以用等式 $g(\theta)=c$ 代替，从 而可以通过 Lagrange 乘子法求解最大值.

\section{2 关于置信限的 EM 算法}

在本节中，对于给定的 $c$, 我们只给出 $\bar{G}(z, c)$ 的计算方法, 关于 $\bar{H}(z, c)$ 的 方法是类似的.

为了得到在条件 $g(\theta)<c$ 下 $G(z, \theta)$ 的最大值, 我们给出一种 EM 算法. 通 常 Newton-Raphson 算法或者格子点方法用来求一个复杂函数的最大值点. 但是 格子点方法要求计算量比较大，特别是对高维参数的情形. 另外函数 $G(z, \theta)$ 是 一个复杂区域上的积分或者求和，形式比较复杂，从而 Newton-Raphson 算法难 于实现. 利用 $\mathrm{EM}$ 算法给定 $\theta$ 的当前值 $\theta^{(t)}$, 用一个相对简单的函数求解下一步 的值 $\theta^{(t+1)}$. 这个想法来自文献 [8]. $\bar{G}(z, c)$ 是概率函数 $G(z, \theta)$ 的最大值. 如果 $G(z, \theta)$ 能够看作一个随机变量的似然函数，则 $\mathrm{EM}$ 算法就能够使用. 按照定义 (1.3), $G(z, \theta)=P_{\theta}(Z \succeq z)=P_{\theta}(I(Z \succeq z)=1)$, 这里 $I(\cdot)$ 为指示函数, 所以概率 $G(z, \theta)$ 可以看作随机变量 $I(Z \succeq z)$ 的似然函数当 $I(Z \succeq z)=1$ 时的值. 下面给 出的是这个特殊似然函数求解最大值的 EM 算法.

为了使得这个方法应用面更加广泛，假定观测数据 $z$ 可能是不完全数据， 它只是完全数据 $y$ 的一个函数, 比如 $z=\mathcal{M}(y)$. 特别地, 如果 $z$ 本身是完全的, 则 $z=y$.

设 $f(y \mid \theta)$ 是 $y$ 在某个测度 $\mu(y)$ 下的似然函数. 从一个初始值 $\theta^{(0)} \in \Theta$ 出 发, 寻找 $\bar{G}(z, c)$ 的算法由重复下面两步构成:

E 步. 给定当前的值 $\theta^{(t)}(t=0,1, \cdots)$, 对于 $y$ 的对数似然函数 $L(\theta \mid Y)=$ $\log f(Y \mid \theta)$ 求条件期望:

$$
Q\left(\theta \mid \theta^{(t)}\right)=E_{\theta^{(t)}}(L(\theta \mid Y) \mid Z \succeq z) .
$$


$\mathbf{M}$ 步. 通过求取 $Q\left(\theta \mid \theta^{(t)}\right)$ 的最大值决定 $\theta^{(t+1)}$ :

$$
Q\left(\theta^{(t+1)} \mid \theta^{(t)}\right) \geqslant Q\left(\theta \mid \theta^{(t)}\right), \quad g(\theta)<c, \theta \in \Theta .
$$

根据 $\mathrm{EM}$ 算法的一般理论有下面的命题 2.1. 序列 $\left\{G\left(z, \theta^{(t)}\right), t=1,2, \cdots\right\}$ 是 递增的. 这样, 如果在若干循环之后 $\theta^{(t)}$ 是稳定的, 则令 $\bar{G}(z, c) \doteq G\left(z, \theta^{(t)}\right)$. 另 外，也许有多个局部极值存在，这样要重复执行 EM 算法多次并且每次选择不同 的初值 $\theta^{(0)}$, 最大的稳定值就可以作为 $\bar{G}(z, c)$.

\section{命题 2.1}

$$
G\left(z, \theta^{(t+1)}\right) \geqslant G\left(z, \theta^{(t)}\right), \quad t=1,2, \cdots .
$$

证 事实上, 递增的性质可以从下面的式子得到:

$$
\begin{aligned}
G\left(z, \theta^{(t+1)}\right)-G\left(z, \theta^{(t)}\right) & =\int_{M(y) \succeq z}\left[f\left(y \mid \theta^{(t+1)}\right)-f\left(y \mid \theta^{(t)}\right)\right] \mu(d y) \\
& \geqslant \int_{M(y) \succeq z}\left[\log f\left(y \mid \theta^{(t+1)}\right)-\log f\left(y \mid \theta^{(t)}\right)\right] f\left(y \mid \theta^{(t)}\right) \mu(d y) \\
& =\left(Q\left(\theta^{(t+1)} \mid \theta^{(t)}\right)-Q\left(\theta \mid \theta^{(t)}\right)\right) G\left(z, \theta^{(t)}\right) \\
& \geqslant 0 .
\end{aligned}
$$

第 1 个不等号成立是因为不等式 $b-a \geqslant(\log b-\log a) a$ 对于所有的 $b>0$ 和 $a>0$ 都成立.

\section{3 例子}

\section{1 指数单元串联系统}

假定一个串联系统由 $m$ 个单元组成, 每个单元寿命都服从指数分布. 第 $i$ 个单元寿命的分布函数为

$$
F\left(x, \lambda_{i}\right)=1-\exp \left(-\lambda_{i} x\right), \quad x>0, \lambda_{i}>0,
$$

这里 $\lambda_{i}$ 称为第 $i$ 个单元的失效率 $(i=1,2, \cdots, m)$, 则整个串联系统服从指数分布

$$
F(x, \lambda)=1-\exp (-\lambda x), \quad x>0,
$$

其中 $\lambda=\sum_{i=1}^{m} \lambda_{i}$ 是整个串联系统的失效率.

对于每个组件, 观测到的是 $\mathrm{I}$ 型删失数据, 即存在一个预先给定的正数 $T_{i}$, 使 得能够观测到 $z_{i j}=\min \left(x_{i j}, T_{i}\right)$ 和 $\delta_{i j}=I\left(x_{i j \leqslant T_{i}}\right)$, 这里 $x_{i j}(i=1,2, \cdots, m, j=$ $1,2, \cdots, n_{i}$ 是组件 $i$ 的第 $j$ 个试验个体的真正寿命, $I(\cdot)$ 是指示函数，则完全统 计量是 $\left(z_{1}, z_{2}, \cdots, z_{m}, r_{1}, r_{2}, \cdots, r_{m}\right)$, 这里 $z_{i}=\sum_{j=1}^{n_{i}} z_{i j}, r_{i}=\sum_{j=1}^{n_{i}} \delta_{i j}$.

我们现在的兴趣在于根据删失数据 $\left(z_{1}, z_{2}, \cdots, z_{m}, r_{1}, r_{2}, \cdots, r_{m}\right)$ 给出系统失 效率 $\lambda$ 的置信上限. 这个问题涉及 $m$ 维参数 $\left(\lambda_{1}, \lambda_{2}, \cdots, \lambda_{m}\right)$ 和删失数据.

对数似然函数为

$$
L\left(\lambda_{1}, \lambda_{2}, \cdots, \lambda_{m}\right)=\sum_{i=1}^{m}\left(r_{i} \log \lambda_{i}-\lambda z_{i}\right) .
$$


$\lambda$ 的最大似然估计为

$$
\hat{\lambda}=\frac{r_{1}}{z_{1}}+\frac{r_{2}}{z_{2}}+\cdots+\frac{r_{m}}{z_{m}} .
$$

对于 $u \geqslant 0$, 设

$$
\begin{gathered}
H\left(u, \lambda_{1}, \lambda_{2}, \cdots, \lambda_{m}\right)=P_{\lambda_{1}, \lambda_{2}, \cdots, \lambda_{m}}(\hat{\lambda}>u), \\
\bar{\lambda}(u)=\sup \left\{\lambda=\sum_{i=1}^{m} \lambda_{i}: H\left(u, \lambda_{1}, \lambda_{2}, \cdots, \lambda_{m}\right)<1-\alpha, \lambda_{i}>0, i=1, \cdots, m\right\} .
\end{gathered}
$$

根据引理 1.1 可知 $\lambda$ 的置信上限为 $\bar{\lambda}(\hat{\lambda})$. 由 (1.8) 式得到

$$
\begin{aligned}
\bar{H}(u, c) & =\sup \left\{1-H\left(u, \lambda_{1}, \lambda_{2}, \cdots, \lambda_{m}\right): \sum_{i=1}^{m} \lambda_{i}>c\right\} \\
& =\sup \left\{P_{\lambda_{1}, \cdots, \lambda_{m}}(\hat{\lambda} \leqslant u): \sum_{i=1}^{m} \lambda_{i}>c\right\} \\
& =\sup \left\{P_{\lambda_{1}, \cdots, \lambda_{m}}(\hat{\lambda} \leqslant u): \sum_{i=1}^{m} \lambda_{i}=c\right\} .
\end{aligned}
$$

最后一个等式的成立是由于 $P_{\lambda_{1}, \cdots, \lambda_{m}}(\hat{\lambda} \leqslant u)$ 和 $\sum_{i=1}^{m} \lambda_{i}$ 关于每一个 $\lambda_{i}$ 的连续性 和单调性.

为了计算 $\bar{H}(u, c)$, 我们使用 $\mathrm{EM}$ 算法. 给定当前值 $\left(\lambda_{1}^{(t)}, \lambda_{2}^{(t)}, \cdots, \lambda_{m}^{(t)}\right)$, 对数 似然函数的条件期望为

$$
Q\left(\lambda_{1}, \lambda_{2}, \cdots, \lambda_{m} \mid \lambda_{1}^{(t)}, \lambda_{2}^{(t)}, \cdots, \lambda_{m}^{(t)}\right)=\sum_{i=1}^{m}\left\{r_{i}^{(t)} \log \lambda_{i}-z_{i}^{(t)} \lambda_{i}\right\},
$$

这里 $r_{i}^{(t)}=E_{\lambda_{1}^{(t)}, \lambda_{2}^{(t)}, \cdots, \lambda_{m}^{(t)}}\left(r_{i} \mid \hat{\lambda} \leqslant u\right), z_{i}^{(t)}=E_{\lambda_{1}^{(t)}, \lambda_{2}^{(t)}, \cdots, \lambda_{m}^{(t)}}\left(z_{i} \mid \hat{\lambda} \leqslant u\right)$.

求 $Q\left(\lambda_{1}, \lambda_{2}, \cdots, \lambda_{m} \mid \lambda_{1}^{(t)}, \lambda_{2}^{(t)}, \cdots, \lambda_{m}^{(t)}\right)$ 在条件 $\sum_{i=1}^{m} \lambda_{i}=c$ 下的最大值, 得到

$$
\lambda_{i}^{(t+1)}=\frac{r_{i}^{(t)}}{z_{i}^{(t)}+k}
$$

这里 $k>-\min \left\{z_{i}^{(t)}: i=1,2, \cdots, m\right\}$ 是 Lagrange 乘子, 它满足

$$
\sum_{i=1}^{m} \frac{r_{i}^{(t)}}{z_{i}^{(t)}+k}=c .
$$

因为 (3.2) 式的左端是 $k$ 的单调减函数, 故可以利用二分法求出数值解. 如果 $t$ 足 够大并且序列 $\left(\lambda_{1}^{(t)}, \lambda_{2}^{(t)}, \cdots, \lambda_{m}^{(t)}\right)$ 稳定, 则设 $\bar{H}(u, c) \doteq H\left(u, \lambda_{1}^{(t)}, \lambda_{2}^{(t)}, \cdots, \lambda_{m}^{(t)}\right)$. 利 用 $\bar{H}(u, c)$ 关于 $c$ 的单调性和连续性, 再由二分法可以求出下面方程的数值解:

$$
\bar{H}(u, c)=\alpha .
$$

由定理 1.1 我们知道置信上限 $\bar{\lambda}(\hat{\lambda})$ 恰好就是这个方程的根.

现在考虑一个由 Nelson ${ }^{[9]}$ 和 Lawless ${ }^{[10]}$ 研究过的实际数据集. 在寿命试验 中, 某种电绝缘液的 76 个试验品分别放入 7 种不同电压水平下做试验, 记录到 每个试验件从开始到失效的时间长度. 我们现在利用这些寿命试验数据作为串 
联系统的 7 个不同组件的试验数据, 7 个组件对应 7 种不同的电压水平. 试验件 的寿命假定服从指数分布，对于不同的电压具有不同的平均寿命. 在这个数据 集中，没有删失数据. 仿照 Jend 等 ${ }^{[11]}$ 处理删失数据的例子所使用的方法，我们 给定试验截止时间 $T_{i} \equiv 20(i=1,2, \cdots, m)$. 我们将摘要数据列于表 1 .

表 1 电绝缘液实验数据摘要

\begin{tabular}{cccccccc}
\hline & 组件 1 & 组件 2 & 组件 3 & 组件 4 & 组件 5 & 组件 6 & 组件 7 \\
\hline$n_{i}$ & 3 & 5 & 11 & 15 & 19 & 15 & 8 \\
$z_{i}$ & 45.79 & 100.00 & 204.81 & 168.57 & 165.04 & 63.59 & 7.33 \\
$r_{i}$ & 1 & 0 & 2 & 9 & 14 & 14 & 8 \\
\hline
\end{tabular}

由表中数据可以计算得到 $\lambda$ 的极大似然估计是

$$
\hat{\lambda}=\frac{1}{45.79}+\frac{0}{100.00}+\cdots+\frac{8}{7.33}=1.4818 .
$$

设 $\alpha=0.2$, 由所建议的方法得到 $\bar{\lambda}(\hat{\lambda})=2.1130$. 初始值 $\left(\lambda_{1}^{(0)}, \lambda_{2}^{(0)}, \cdots, \lambda_{m}^{(0)}\right)$ 被随 机选取的 $\mathrm{EM}$ 算法重复 10 次后，最大的稳定值作为 $\bar{H}(u, c)$. 有时 $\bar{H}(u, c)$ 在集合

$$
\left\{\left(\lambda_{1}, \lambda_{2}, \cdots, \lambda_{m}\right): \lambda_{1}+\lambda_{2}+\cdots+\lambda_{m}=c, \lambda_{i} \geqslant 0, i=1,2, \cdots, m\right\}
$$

的边界点达到, 有时在内部达到, 这可以对 $m=2$ 的情形通过数值结果验证.

\section{2 两个比例的差}

在医学和工业中，为了评估某项新措施的效果，经常要比较两组中成功的 比例 (可参考文献 [12,13]), 通常给出的是基于大样本的置信限, 这在小样本的情 形下是不准确的.

用严格的语言来说，设 $S_{1}$ 和 $S_{2}$ 是两个独立的二项分布的随机变量，分别 具有参数 $\left(n_{1}, p_{1}\right)$ 和 $\left(n_{2}, p_{2}\right)$. 下面给出差 $p_{1}-p_{2}$ 的置信下限的 EM 算法:

定义 $\left(s_{1}, s_{2}\right) \succeq\left(s_{1}^{\prime}, s_{2}^{\prime}\right)$ 当且仅当

$$
\frac{s_{1}}{n_{1}}-\frac{s_{2}}{n_{2}} \geqslant \frac{s_{1}^{\prime}}{n_{1}}-\frac{s_{2}^{\prime}}{n_{2}} .
$$

设

$$
\begin{gathered}
G\left(s_{1}, s_{2}, p_{1}, p_{2}\right)=P_{p_{1} p_{2}}\left(\frac{S_{1}}{n_{1}}-\frac{S_{2}}{n_{2}} \geqslant \frac{s_{1}}{n_{1}}-\frac{s_{2}}{n_{2}}\right), \\
\bar{G}\left(s_{1}, s_{2}, c\right)=\sup \left\{G\left(s_{1}, s_{2}, p_{1}, p_{2}\right): p_{1}-p_{2}<c, 0 \leqslant p_{1} \leqslant 1,0 \leqslant p_{2} \leqslant 1\right\}, \\
\underline{g}\left(s_{1}, s_{2}\right)=\sup \left\{c: \bar{G}\left(s_{1}, s_{2}, c\right)<\alpha, c>0\right\} .
\end{gathered}
$$

由前面给出的理论, $g\left(s_{1}, s_{2}\right)$ 是 $p_{1}-p_{2}$ 的置信下限, 置信水平为 $1-\alpha$.

给定当前值 $p^{(t)}=\left(p_{1}^{(t)}, p_{2}^{(t)}\right)$, $\mathrm{E}$ 步由 (2.1) 式给出. 经过简单的计算知 $\mathrm{M}$ 步 归结为求解方程

$$
\begin{aligned}
& \frac{E_{p_{(t)}}\left(S_{1} \mid S \succeq s\right)}{p_{2}+c}-\frac{n_{1}-E_{p_{(t)}}\left(S_{1} \mid S \succeq s\right)}{1-p_{2}-c} \\
+ & \frac{E_{p_{(t)}}\left(S_{2} \mid S \succeq s\right)}{p_{2}}-\frac{n_{1}-E_{p_{(t)}}\left(S_{2} \mid S \succeq s\right)}{1-p_{2}}=0,
\end{aligned}
$$




$$
\max \{0,-c\}<p_{2}<\min \{1,1-c\}, \quad p_{1}=p_{2}+c .
$$

由于上面方程的左端是 $p_{2}$ 的单调减函数, 并且当 $p_{2} \rightarrow \max \{0,-c\}^{+}$时趋于 $+\infty$, 当 $p_{2} \rightarrow \min \{1,1-c\}^{-}$时趋于 $-\infty$, 故方程的数值解容易找到.

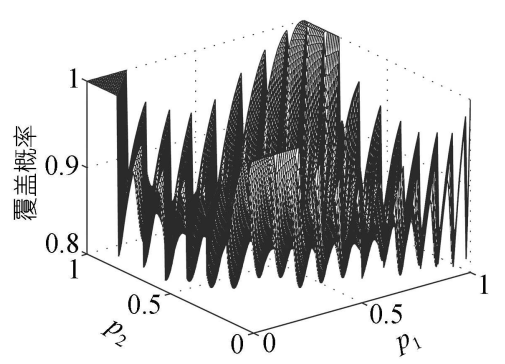

图 1 覆盖概率

对于 $n_{1}=8, n_{2}=10, \alpha=0.2$ 的情形, 我们在 $p_{1}, p_{2}=0.01,0.02, \cdots, 1$ 时计算了覆 盖概率

$$
\gamma=P_{p_{1} p_{2}}\left(p_{1}-p_{2} \geqslant \underline{g}\left(s_{1}, s_{2}\right)\right) .
$$

图 1 给出了曲面 $\left(p_{1}, p_{2}, \gamma\right)$. 可以看出这个置 信下限的实际水平就是它的名义水平 0.80 . 我 们看到曲面多次达到底部, 所以这个置信下 限表现很好.

\subsection{Gamma 随机变量的均值}

设 $Y_{1}, Y_{2}, \cdots, Y_{n}$ 是独立同分布随机变量, 具有 Gamma 分布密度

$$
f(y)=\frac{y^{\gamma-1} \exp (-y / \beta)}{\beta^{\gamma} \Gamma(\gamma)} \quad(\gamma>0, \beta>0) .
$$

这个模型由 Meng 等 ${ }^{[7]}$ 用来说明求最大似然估计的期望条件最大算法 (ECM), 我 们现在用来得到平均寿命 $E Y_{1}=\gamma \beta$ 的置信下限. 在仔细推导之前我们给出一个 结果. 基于 $\gamma \beta$ 最大似然估计 $\bar{Y}^{(n)}\left(=(1 / n) \sum_{i=1}^{n} Y_{i}\right)$, Buehler 置信下限是 $\bar{Y}^{(n)}$ 和 一个系数的乘积, 这个系数仅仅依赖于置信水平而与样本量和观测值无关. 另 外, 这个系数仍然可以通过 EM 算法得到, 因为它是一个特例的置信下限值. 在 本节的最后对于几种置信水平给出计算结果. 下面进行详细讨论：

对于观测值 $y=\left(y_{1}, y_{2}, \cdots, y_{n}\right)$, 对数似然函数是

$$
L(\gamma, \beta \mid Y)=(\gamma-1) \sum_{i=1}^{n} \log y_{i}-\frac{1}{\beta} \sum_{i=1}^{n} y_{i}-n\{\gamma \log \beta+\log \Gamma(\gamma)\} .
$$

可以证明 $E Y_{1}$ 的最大似然估计是 $\bar{y}^{(n)}\left(=\sum_{i=1}^{n} y_{i} / n\right)$. 为了得到它的置信下限, 我 们定义样本空间的序 $\succeq$, 使得 $Y^{(n)}=\left(Y_{1}, \cdots, Y_{n}\right) \succeq y^{(n)}=\left(y_{1}, \cdots, y_{n}\right)$ 当且仅当 $\bar{Y}^{(n)} \geqslant \bar{y}^{(n)}$. 对于任意 $u>0$, 设

$$
\begin{gathered}
G_{n}(u, \gamma, \beta)=P_{\gamma \beta}\left(\frac{1}{n} \sum_{i=1}^{n} Y_{i} \geqslant u\right), \\
\underline{g_{n}}(u)=\inf \left\{\gamma \beta: G_{n}(u, \gamma, \beta)>\alpha, \gamma>0, \beta>0\right\},
\end{gathered}
$$

这里 $P_{\gamma \beta}(A)$ 表示参数为 $\gamma$ 和 $\beta$ 时事件 $A$ 的概率. 由引理 1.1 知 $\underline{g_{n}}\left(\bar{Y}^{(n)}\right)$ 是 $\gamma \beta$ 的置信下限, 置信水平为 $1-\alpha$.

\section{命题 3.1}

$$
\underline{g_{n}}\left(\bar{Y}^{(n)}\right)=\bar{Y}^{(n)} h(\alpha),
$$

这里

$$
h(\alpha)=\underline{g_{1}}(1)=\inf \left\{\gamma \beta: G_{1}(1, \gamma, \beta)>\alpha, \gamma>0, \beta>0\right\},
$$




$$
G_{1}(1, \gamma, \beta)=P_{\gamma \beta}\left(Y_{1} \geqslant 1\right) .
$$

证 因为和 $\sum_{i=1}^{n} Y_{i}$ 具有 Gamma 分布参数 $n \gamma, \beta$, 故有等式

$$
G_{n}(u, \gamma, \beta)=G_{1}(n u, n \gamma, \beta) .
$$

由于 $\beta$ 是尺度参数, 可以证明等式

$$
G_{1}(n u, n \gamma, \beta)=G_{1}\left(1, \gamma, \frac{\beta}{n u}\right),
$$

所以

$$
\begin{aligned}
\underline{g_{n}}(u) & =\inf \left\{\gamma \beta: G_{n}(u, \gamma, \beta)>\alpha, \gamma>0, \beta>0\right\} \\
& =\inf \left\{\gamma \beta: G_{1}\left(1, n \gamma, \frac{\beta}{n u}\right)>\alpha, \gamma>0, \beta>0\right\} \\
& =u \inf \left\{(n \gamma)\left(\frac{\beta}{n u}\right): G_{1}\left(1, n \gamma, \frac{\beta}{n u}\right)>\alpha \gamma>0, \beta>0\right\}, \\
& =u \inf \left\{\gamma^{*} \beta^{*}: G_{1}\left(1, \gamma^{*}, \beta^{*}\right)>\alpha, \gamma^{*}>0, \beta^{*}>0\right\} \\
& =u \inf \left\{\gamma \beta: G_{1}(1, \gamma, \beta)>\alpha, \gamma>0, \beta>0\right\} \\
& =u h(\alpha) .
\end{aligned}
$$

从而 (3.7) 式成立. 证毕.

为了得到 $h(\alpha)$, 我们使用 $\mathrm{EM}$ 算法. 对于任意 $c>0$, 设

$$
\bar{G}(c)=\sup \left\{G_{1}(1, \gamma, \beta): \gamma \beta<c, \gamma>0, \beta>0\right\} .
$$

利用定理 1.1 , 我们知道下面的等式成立:

$$
h(\alpha)=\sup \{c: \bar{G}(c)<\alpha, c>0\} .
$$

因为 $G_{1}(1, \gamma, \beta)$ 关于 $\beta$ 单调递减并且连续, 故可以证明 (3.9) 式中的严格不等式 $\gamma \beta<c$ 可以由等式 $\gamma \beta=c$ 代替, 即

$$
\bar{G}(c)=\sup \left\{G_{1}(1, \gamma, \beta): \gamma \beta=c, \gamma>0, \beta>0\right\} .
$$

对于给定的 $c>0$, 我们现在描述得到 $\bar{G}(c)$ 的 $\mathrm{EM}$ 算法. 设

$$
\begin{aligned}
Q\left(\gamma, \beta \mid \gamma^{(t)}, \beta^{(t)}\right)= & (\gamma-1) E_{\gamma^{(t)} \beta^{(t)}}\left(\log Y_{1} \mid Y_{1} \geqslant 1\right) \\
& -\frac{1}{\beta} E_{\gamma^{(t)} \beta^{(t)}}\left(Y_{1} \mid Y_{1} \geqslant 1\right)-\{\gamma \log \beta+\log \Gamma(\gamma)\} .
\end{aligned}
$$

根据 $\mathrm{EM}$ 算法, 需要寻找 $\left(\alpha^{(t+1)}, \beta^{(t+1)}\right)$, 使得

$$
Q\left(\gamma^{(t+1)}, \beta^{(t+1)} \mid \gamma^{(t)}, \beta^{(t)}\right) \geqslant Q\left(\gamma, \beta \mid \gamma^{(t)}, \beta^{(t)}\right), \quad \forall \gamma \beta=c, \gamma>0, \beta>0 .
$$

设 $Q\left(\gamma, c / \gamma \mid \gamma^{(t)}, \beta^{(t)}\right)$ 关于 $\gamma$ 的导数为 0 , 可以得到方程

$$
\frac{\Gamma^{\prime}(\gamma)}{\Gamma(\gamma)}-\log \gamma=E_{\gamma^{(t)} \beta^{(t)}}\left(\log Y_{1} \mid Y_{1} \geqslant 1\right)-\frac{1}{c} E_{\gamma^{(t)} \beta^{(t)}}\left(Y_{1} \mid Y_{1} \geqslant 1\right)-\log c+1 .
$$

可以证明方程 (3.13) 的左端是 $\gamma$ 的单调增函数，因此方程 (3.13) 的数值解容易得 到. 方程的根记为 $\gamma^{(t+1)}$, 则 $\beta^{(t+1)}=c / \gamma^{(t+1)}$.

因此给定 $\alpha \in(0,1), c>0$ 和初值 $\left(\gamma^{(0)}, \beta^{(0)}\right)$, 重复求解方程 (3.13) 直到序列 $\left(\gamma^{(t)}, \beta^{(t)}\right)$ 稳定. 设稳定值 $G_{1}\left(1, \gamma^{(t)}, \beta^{(t)}\right)$ 作为 $\bar{G}(c)$ 的近似, 即

$$
\bar{G}(c) \doteq G_{1}\left(1, \gamma^{(t)}, \beta^{(t)}\right),
$$


因此 $h(\alpha)$ 可以由 (3.10) 式利用二分法求解. 对于 $\alpha=0.05,0.10,0.15,0.20$, 分别 可以得到结果 $h(\alpha)=0.171615,0.330617,0.476017,0.606673$. 在这个例子中, 初值 的选取对结果 $\bar{G}(c)$ 没有很大的影响.

\section{4 讨论}

EM 算法对于寻找不完全数据情形下的极大似然估计是一个有用的方法. 本 文通过一个变换将 EM 算法用于求解 Buehler 置信限. 特别对于小样本情形, 此 方法很有用. 这个方法在原则上与 EM 算法求取极大似然估计有相同的适用范 围. 另外, 所有对 EM 算法的推广 (如 ECME, AECM 等) 也可以用于求解 Buehler 置信限.

然而 EM 算法本身的缺点仍然存在. 对于有些情形, 算法可能收玫非常慢. 尽管在理论上这个方法可以用到非常一般的模型，但是 EM 算法的收敛速度以 及计算量都限制了它的应用范围. 这些方法还有许多值得改进的地方.

致谢 作者感谢评审者对本文提出的有益建议.

\section{参考文献}

1 Buehler R J. Confidence interval for the product of two Binomial parameters. J Amer Statist Assoc, 1957, 52(2): 482 493

2 Jobe J M, David H T. Buehler confidence bounds for a reliability-maintainability measure. Technometrics, 1992, 34(2): 214 222

3 陈家鼎. 样本空间的序与参数的置信限. 数学进展, 1993, 22(6): 542 552

4 Kabaila P. Better Buehler confidence limits. Statistics \& Probability Letters, 2001, 52(2): 145 154

5 Dempster A P. Laird N M, Rubin D B. Maximum likelihood from incomplete data via EM algorithm (with discussion). J R Statist Soc B, 1977, 39(1): 1 38

6 Liu C, Rubin D B. The ECME algorithm: a simple extension of EM and ECM with fast monotone convergence. Biometrika, 1994, 81(4): 633 648

7 Meng X L, Dyk V D. The EM algorithm-an old folk-song sung to a fast new tune. J R Statist Soc B, 1997, 59(3): $511 \sim 567$

8 房祥忠, 陈家鼎. EM 算法在假设检验中的应用. 中国科学, A 辑, 2003, 33(2): 180 184

9 Nelson W B. Graphical analysis of accelerated life test data with the inverse power law models. IEEE Trans Reliab, 1972, R-21: 2 11

10 Lawless J F. Statistical models and methods for lifetime data. 2nd ed. New Jersey: A John Wiley and Sons, 2003. 3

11 Jend S L. William Q M. Comparisons of approximate confidence interval procedures for type I censored data. Technometrics, 2000, 42(2): 135 148

12 Fagan T. Exact $95 \%$ confidence interval for differences in binomial proportions. Computers in Biology and Medicine, 1999, 29(1): 89 87

13 Wallenstein S. A non-iterative accurate asymptotic confidence interval for the difference between two proportions. Statistics in Medicine, 1997, 16(12): 1329 1336 